\title{
Biomedical Informatics Research and Education at the EuroMISE Center
}

\author{
J.Zvarova \\ European Centre for Medical Informatics, Statistics and Epidemiology, Institute of Computer Science \\ AS CR, Prague, Gech Republic
}

\section{Introduction}

The European Centre for Medical Informatics, Statistics and Epidemiology (EuroMISE Centre) was set up in April 1994 as the joint Teaching and Research Centre of Charles University and the Academy of Sciences of the Czech Republic with the support of European projects. In the year 2000, the EuroMISE Centre was enlarged with three additional institutions (University of Economics in Prague, General University Hospital in Prague and Municipal Hospital in Caslav). Nowadays, the EuroMISE Centre creates an interdisciplinary workplace that continually, and on the European level, focuses on the research and development on information and knowledge technologies for the improvement of healthcare and higher education. Through the research cooperation, the EuroMISE Centre (http:// www.euromise.cz) suggests publicly available tools and methods that allow the application of new technologies for various healthcare organizations and citizens with a special focus on the Czech national environment. It provides a platform for a higher level of research cooperation with the usage of the most up-to-date information and communication technologies and a wide spectrum of clinical and genetic data and knowledge. Some activities in biomedical research, higher education and knowledge dissemination are further described in more details.

\section{Biomedical Informatics - Education and Knowledge Dissemination}

\subsection{EuroMISE Courses in English}

University education is the basis for research and development. This is true for all areas of business and also for healthcare. In Europe, the curricula still differ in content, structure and style. Supporting programmes of the European Commission such as ERASMUS and TEMPUS were created to assist in the transfer of knowledge among European countries. In the period from 1993-1995 the Joint European Project "Education in the Methodology Field of Health Care" of the TEMPUS programme was running giving the assistance of European Union countries to Central and Eastern European countries. More detailed information about the aims of the project are given in [1]. The project was running under the acronym EuroMISE (European education in Medical Informatics, Statistics and Epidemiology) with the participation of eleven leading universities and research institutions from EU countries [2]. Training and education in EuroMISE consisted of three overlapping methodological branches: medical informatics, medical statistics and epidemiology. One of the main goals of the EuroMISE project was to create a teaching network for higher education 
in the multidisciplinary field of health care and to create basic conditions to multiply knowledge and skills received in the courses. During the year 1993 the EuroMISE courses in English were developed and in April 1994 the EuroMISE Center, as the joint teaching and research centre of Charles University in Prague and the Academy of Sciences of the Czech Republic, was set up. In 1994 and 1995, EuroMISE courses were taught by university professors and senior researchers from EU universities and research institutions. More than 70 certificates were passed in the Charles University Aula Magna for successful completion of the European EuroMISE courses in 1994 and 1995 by participants from the Czech Republic and other Central and Eastern European countries (Bosnia and Herzegovina, Bulgaria, Croatia, Hungary, Poland, Romania, and Slovenia). In 1996 and 1997, postgraduate and continuing education EuroMISE courses in English, which started in the frame of the European cooperation via the EuroMISE project, were further running with the great support of participating EU institutions (Erasmus University, Rotterdam and Limburg University, Maastricht, The Netherlands, Ruprecht-Karls University, Heidelberg, Phillips University, Marburg and MEDIS Institute, Neuherberg, Germany, University of Manchester, Institute of Science and Technology, United Kingdom, Catholic University of Leuven and Free University of Brussels, Belgium, University of Bordeaux II, France, Aristotle University of Thessaloniki, Greece, Trinity College Dublin, Ireland). The support of the EU institutions made it possible to run the EuroMISE courses free of charge for students from different European countries. In 1996 and 1997, more than 50 certificates were awarded for the successful completion of the EuroMISE courses. The EuroMISE courses have opened the challenge to improve the training opportunities in the field of medical informatics, statistics and epidemiology in Central and Eastern European countries and contributed to positive changes in the health care sector.

\subsection{Information Technologies for Education and Training}

The main aim of the project of the 4th Framework Programme of EU countries IT EDUCTRA (Information Technologies EDUcation and TRAining) running in years 1996-1998 was to contribute to the propagation of knowledge on how to utilize information technologies in teaching and training in health care. The project associated 20 universities, research institutions and companies. One of the associated partners in the project was also the EuroMISE Centre of Charles University and the Academy of Sciences. To reach determined goals, the project firstly summarized knowledge gained in other European programmes, e.g. AIM, ERASMUS, DELTA or TEMPUS [3]. Consequently, the main directions for designing teaching materials were proposed. It was found that the highest priority was given to the topics such as electronic communication, electronic patient records, data protection and security, computer supported diagnostics and management of health care quality. Ten teaching topics were designed on the basis of the analyses of needs in teaching in healthcare. Suitable teaching aids, e.g. pictures, tables and graphs in an electronic form, examples with results of their solution or surveys of a contemporary state of problems in the world were created in the project. Teaching materials were first developed in English. Gradually their versions in German, French, Spanish, and other European languages were created.
The EuroMISE Centre of Charles University and the Academy of Sciences were involved in the translation of selected teaching materials into the Czech language. The project gradually developed mechanisms on how to train more than 5000 European professionals in health care and how to spread these courses into other institutions in order to multiply the number of graduates in a cascade effect. An innovation in using modern information technologies in teaching was testing possibilities of a videoconferencing system by ISDN technology, which was a real novelty for the Czech Republic at that time. Teaching materials on CD were developed during the project and CDs were available free of charge. All materials were in English and presentations were also accessible in Czech versions [4]. The ExaMe system for the evaluation of targeted knowledge has been in development since 1998 [5]. The idea of the system is based on multiplechoice questions, but with no prior restrictions on the number of selected answers. The only restriction is that at least one answer is correct and at least one wrong. This new idea has led to new concepts of standardization of test results and many new theoretical developments. The ExaMe evaluation system is an important part of education and training in the EuroMISE Center. The ubiquity of the Internet and its World Wide Web applications made it possible to realize the new educational goals in an innovative and creative way. New features of the ExaMe evaluation system are described in [6] in more details.

\subsection{Doctoral Degree - Ph.D. in Biomedical Informatics}

Charles University, in comparison with other universities in the Czech Republic, 
gives the largest number of courses and together with the Academy of Sciences of the Czech Republic has the most extensive research in different medical specializations. Nowadays, science and higher education have a closer relationship and increasing interdisciplinary cooperation. This cooperation offers enrichment of different disciplines with diverse knowledge that might contribute with new insights to solve existing problems, e.g. that of over specialization in any given field, including its methodology and terminology. One of the most important tasks is to strengthen educational activities in this field and to create the basic assumptions for penetration of biomedical informatics methodologies to the field of biomedical research and health care. The agreement on cooperation of Charles University in Prague and the Academy of Sciences of the Czech Republic in postgraduate doctoral studies was signed on April $23^{\text {rd }}, 1997$. The main goal of this agreement has been cooperation in the development and running of joint education and training of young researchers. The conceptually unified system for postgraduate doctoral studies has been established for this purpose. In accordance with Czech law no.172/90 Coll. on universities, the postgraduate doctoral studies are organized by Charles University in Prague. Therefore the graduates entering the postgraduate doctoral studies are students of faculties of Charles University in Prague. The board is composed of leading scientists from both Charles University and the Academy of Sciences of the Czech Republic. The system of postgraduate doctoral studies in biomedicine has been opened based on this agreement. Biomedical informatics covers the fields of bioinformatics, medical and health informatics. The synergy between bioinformatics and medical informatics facilitating genomic medicine for future health care was deeply studied in [7]. There are now 19 boards of scientific disciplines in postgraduate doctoral studies in biomedicine, one of them is the scientific board of Biomedical Informatics accredited in the year 2002 for teaching in Czech and accredited in the year 2005 for teaching in English as well. After successfully passing an entrance exam, students are entering studies in biomedical informatics as the doctoral students of the First Faculty of Medicine of Charles University. The scientific board of Biomedical Informatics accepts about 10 new students each year and the first student successfully graduated from the Biomedical Informatics doctoral program in the year 2004. During their studies the doctoral students have to choose at least two courses from selected scientific disciplines connected with topics of their thesis. Selected courses prepared and given in the EuroMISE Center (Figure 1) are based on the experience from European EuroMISE courses and other international experience in educa- tion and training, e.g. [8], [9], [10], [11]. Based on the experience in the European projects, the EuroMISE Center has started to develop two series of books in Czech in the fields of Biomedical Informatics and Biomedical Statistics. Printed versions of the books are issued by the Carolinum Printing House of Charles University in Prague; their electronic versions are available on the Internet [12], [13], [14], [15]. The first information on biomedical informatics doctoral studies and on the exploration of the electronic textbooks and other tools developed in the EuroMISE Center was presented in [16].

It seems that the Ph.D. studies in biomedical informatics are matching the recent research needs of the Czech Republic. They might help to accelerate the development of interdisciplinary biomedical research to the sound European level. The long-term effect of postgraduate doctoral education in the field of biomedical informatics results in the rapid increase of scientists that are needed in the Czech Republic to

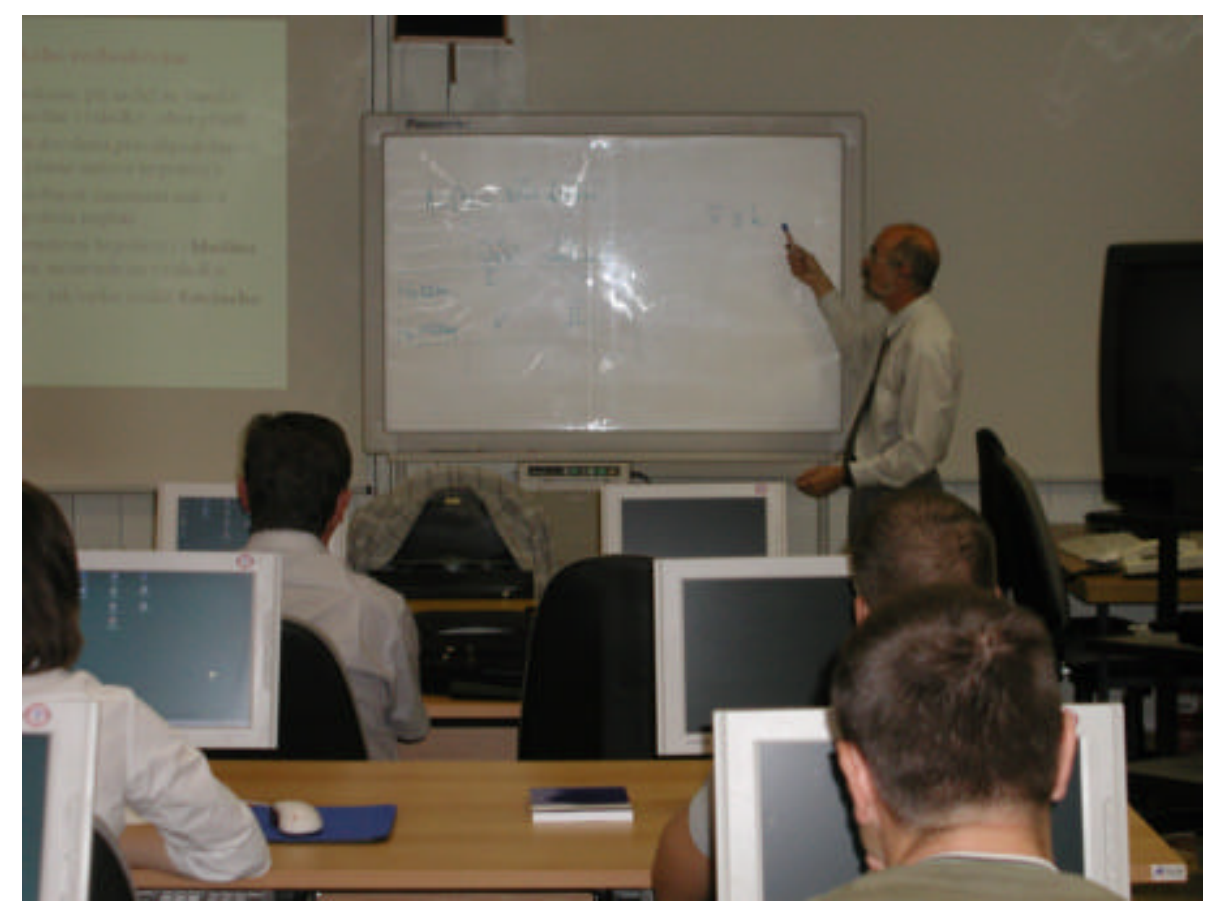

Fig. 1 EuroMISecoursefor dodoral students 
reach the goals of information society in health care.

\subsection{Dissemination of Biomedical Informatics Knowledge}

The PRO-ACCESS project was initiated in the late phase of the 5th Framework Programme as a supporting action running in the years 2002-2004. It focused on the transfer of current concepts in medical telematics to countries remaining in the pre-accession phase The Krakow Centre of Telemedicine in Poland carried out the process of dissemination of up to date approaches to ehealth environment development. One of the cooperating partners in the PROACCESS was the EuroMISE Center. The strategy employed within the PROACCESS project supposed to yield "critical mass" necessary for facilitating the e-health development in this region of Europe. The activities employed to reach this objective included publishing activities, events and trainings as well as intake of solutions from supporting centres, see e.g. [17]. Some results of the PRO-ACCESS project were also presented in the frame of the workshop RTD Potential in Central and Eastern Europe for Building Information Society in Healthcare of the International Joint Meeting EuroMISE 2004 [18]. In April 2004 more than 250 experts from 30 countries gathered in Prague, the Czech Republic, to participate in the International Joint Meeting EuroMISE 2004 that was composed of several scientific events. The working group on "Biomedical Statistics and Information Processing" of the International Medical Informatics Association (IMIA) organized the conference Statistical Methodology in Bioinformatics and Clinical Trials in the frame of the meeting. The working group on Electronic Health Records (EHR) of the European
Federation for Medical Informatics (EFMI) organized the symposium Electronic Health Record, Healthcare Registers and Telemedicine that brought together researchers and health professionals to discuss different approaches to electronic health record development, to examine possibilities of current technologies for telemedicine and to examine healthcare registers as important sources for evidence-based medicine. The symposium Biomedical Informatics and Biomedical Statistics Education was held at the occasion of the $10^{\text {th }}$ anniversary of the EuroMISE Centre and the symposium on Computerized Guidelines and Protocols intended to identify use cases for guideline-base applications in healthcare, pressing issues and promising approaches for developing usable and maintainable vehicles for guideline delivery [19]. Finally, the workshop on the HL7 standard was held in the frame of the Prague meeting.

Apart from educational and teaching activities of the EuroMISE Center on graduate, postgraduate and life-long educational levels, the EuroMISE Center has started to cooperate in a large Czech project focused on the development of the network supporting continuing health telematics education and eHealth. New courses, targeted to the educational needs of the Czech Republic, are under development.

In the year 2005, the EuroMISE Centre started a new initiative to publish the European Journal of Biomedical Informatics on the Internet. The journal provides the opportunity to publish papers not only in original English versions but also in the translated versions of authors' native languages. The new ideas published in the papers are then more accessible to the general public, as not all scientists and physicians are fluent in English. The multilingual versions of papers also help solve problems with terminology.
More information about the European Journal of Biomedical Informatics can be found at http://www.ejbi.org/.

\section{Biomedical Informatics Research}

\subsection{National Program of Research and Development}

The national program of research and development in the Czech Republic was elaborated on in two foresights organized in the years 2001 and 2003. They helped to significantly formulate the content and structure of the key research topics. More then 500 experts independently evaluated various topics and set up their preferences. The detailed SWOT analysis was applied to quantify the results of expert reports and evaluation. The Czech government approved the proposal of the first foresight in April 2003. The research priorities were implemented in the call for proposals opened in February 2004 for the period 2004-2008. Several of the core areas covered by IT disciplines were directly focused on the healthcare issues or on the healthcare domain, where Internet and telemedicine play a very important role. The first foresight showed that the research and development priorities are also focused on different topics of biomedical informatics like electronic health records, medical decision support and telemedicine. Nowadays, the large amounts of genetic and proteomic data offer opportunities for new research targets in biomedical informatics. Within this framework, it is equally important to integrate clinical, genetic and proteomic data by electronic health records. In such an environment, datacentered electronic health records, clinical decision support systems, and image and signal processing techniques and 
tools can be applied to a broader field of information. The EuroMISE Center has developed some of the above mentioned research fields with the support of both European projects and national research projects. We also considered the future exploitation of new information technologies in healthcare [20]. Further in the paper we summarize results based on the structure of research activities of the EuroMISE Center (Figure 2) in the field of biomedical informatics. The multidisciplinary research at the EuroMISE Center moves further towards direct usage in the informational support of diagnostics and therapy, mining of the medical databases while observing all the legal conditions of the Czech law, in customization of healthcare based on the clinical and genetic profile of a patient and to strengthen the prevention of civilization illnesses via the out-patient departments of preventive cardiology.

\subsection{Biomedical Data and Knowledge}

Recently, several trends in biomedicine and health care have been moving towards more extensive use of information technology and particularly knowledge technology. The volume of data stored in extensive and complex electronic databases has been growing constantly. These data describe, in minute details, various aspects of a preventive care, diagnostics and patient therapy, and their analyses allow obtaining new, so far unknown, information. New emerging technologies allow producing results of genetic and biomedical analyses directly in an electronic form. New and constantly developing tools are available, which will make it possible to gain new knowledge from these data that cannot be gained otherwise. There is a growing need to confront the

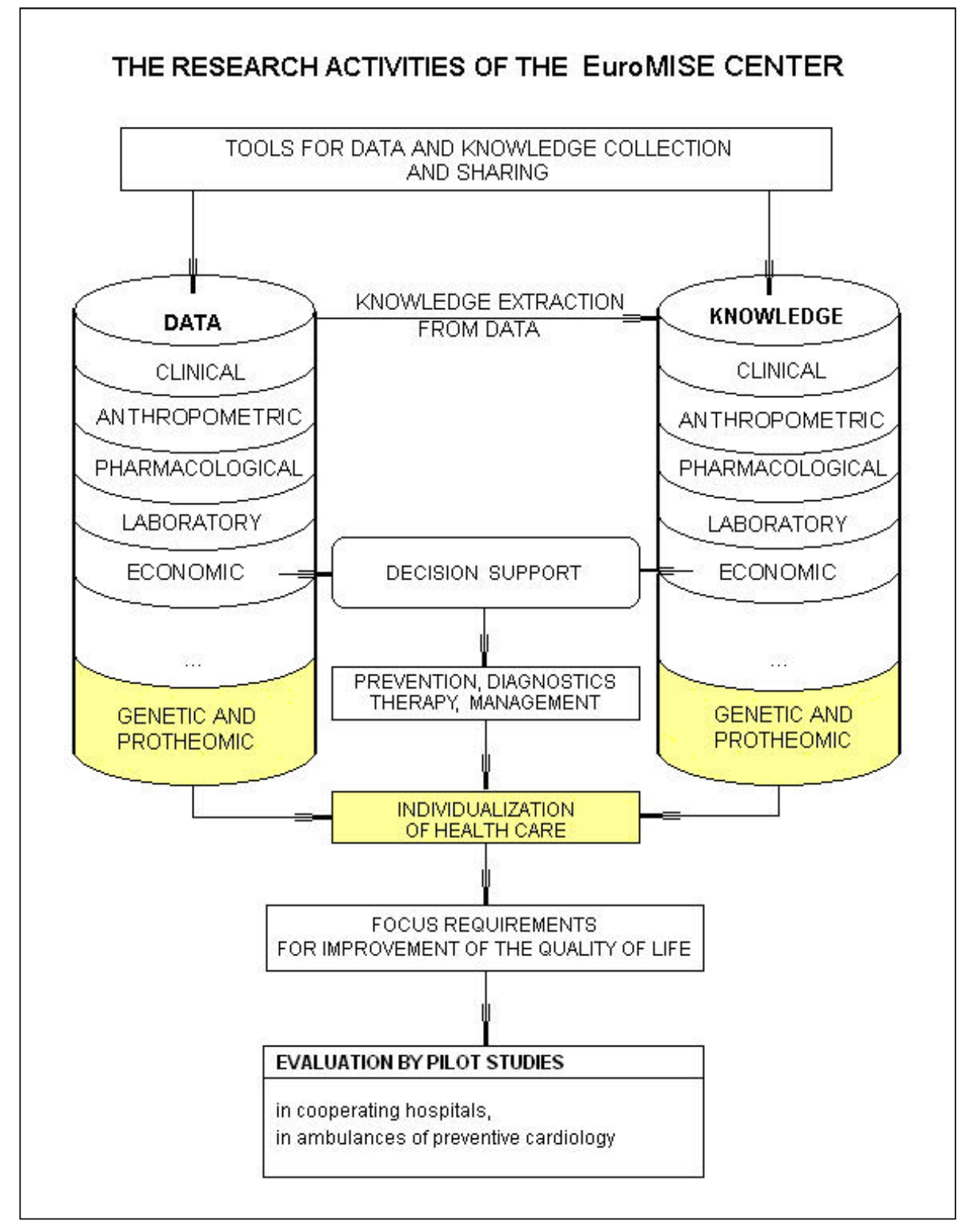

Fig. 2 Main Research Activities of the EuroMISE Center

knowledge gained from the data with other knowledge gained, for instance, through the formalization of medical advice or directly from medical specialists. By combining these kinds of knowledge, a considerable synergic effect can be achieved, for instance, in medical decision support. There is a growing need to efficiently use different forms of knowledge in all fields of medical and healthcare activities at different levels (health centres, hospitals, laboratories, ministries, regional offices, insurance companies, various territorial entities, etc.). There is also a growing conviction that it is possible to improve health care in both quality and economy by using knowledge efficiently.

The EuroMISE Centre has long-standing experience in data mining and applications of advanced statistical approaches, for example in the application of relevant methods to extract knowledge from medical databases. It possesses advanced methods for dimensionality reduction, multidimensional 
models, Bayesian networks and decision support systems. The EuroMISE Center used a large medical database developed in the frame of the twenty year lasting longitudinal study (STULONG) of 1417 middle aged men with risk factors of atherosclerosis. This originated at the Second Department of Internal Medicine of the General University Hospital in Prague for research purposes. Apart from advanced statistical methods, several data mining approaches were used for the data analysis. The results of the analysis contributed to the assessment of mortality risk factors in connection with cardiovascular diseases in the Czech Republic. The Internet presentation of this project http://euromise. vse.cz/stulong-en/index.php includes inter alia an extensive graphical presentation of primary data and results of the detailed analysis. The STULONG data have also been used for the Discovery Challenge contest at the ECML/PKDD 2002, the ECML/PKDD 2003, and the ECML/PKDD 2004 conferences [21]. Other large anonymous medical databases serving research purposes have been developed at the EuroMISE Center. For example, important data and knowledge have been gathered under the guidance of clinicians from the Municipal Hospital of Caslav where the pilot study on acute heart attacks is conducted [22]. From the out-patient departments of preventive cardiology supervised in the frame of the EuroMISE Center by the Municipal Hospital in Caslav and General University Hospital in Prague, anonymous data on healthy controls can be provided for specified research tasks. Biomedical information in different forms is one of the research topics of the EuroMISE Center. Information as the central concept of biomedical informatics was analysed in [23]. Further results of theoretical research on how to use the information theory approach to analyse information in different forms are given in [24] and results on how to measure genetic information, diversity and distance are given in [25]. An important topic concerns information given in the form of free text. In medicine we face this difficult research problem with free text medical records in the Czech language. Some studies in this field were given in papers [26], [27], [28].

\section{3. Eledronic Health Record and Medical Guidelines}

Better quality of the provided health care is also dependent on a good interconnection of the electronic documentation systems to the decision-making and management support systems. Development of advanced techniques of coding, structuring, storing, transmitting and sharing medical data and knowledge also belongs to the research tasks of the EuroMISE Center. One of the important projects in this field, recently realized at the EuroMISE Centre, has been the development of a new system for a structured electronic health record called the MUltimedia Distributed Record [29], [30], [31]. We present the MUDRLite universal solution. It is an easy way to build an electronic health record tailored exactly to your needs. In the first instance, we are deploying MUDRLite to the Neurovascular Department of the Central Military Hospital in Prague. We also started cooperation with the Dental Medicine Department of Charles University, $1^{\text {st }}$ Faculty of Medicine, which should result in spreading MUDRLite among Czech stomatological out-patient departments [32], see (Figure 3). One of the MUDR's version 2 most valued features is that it allows changing the data model dynamically and on the fly [33]. In addition, it supports versioning the data model, so access to the data is possible not only according to the current date, but also according to an arbitrary point in the past. This feature is important for checking whether a treatment conforms to the medical guidelines. It is known that only a small number of physicians use clinical paper-based guidelines during diagnosing and treatment. This problem is caused by the existence of a large amount of relevant guidelines, which are frequently updated. We have experience in formalizing clinical guidelines using the Guideline Interchange Format (GLIF) [34]. In a stage of the GLIF model construction from text guidelines, it is important to find logical and process structures of guidelines, all fundamental parameters and their interrelationships. The result of this cooperation is the graphic GLIF model that corresponds to the text guidelines. The construction stage is the most important and difficult of all stages. Besides, a list of basic and derived parameters is created. Basic parameters represent directly measurable values. Derived parameters are obtained in arithmetical, logical or logical-arithmetical operations above basic parameters. The result is the data model that serves as an interface between the GLIF model and real input data stored in the Electronic Health Record. The GLIF model is graphical so it is necessary to code it in the XML form. In a language form the encoded guidelines consist of a sequence of guideline steps. The GLIF model coded in XML can be used in several types of applications. The GLIF browser offers users a more accessible form of knowledge presentation than classical text. Moreover, it can show explanatory information with different levels of details. It can be used for educating students and as a decision support system in medical practice. The Reminder system checks if input data 


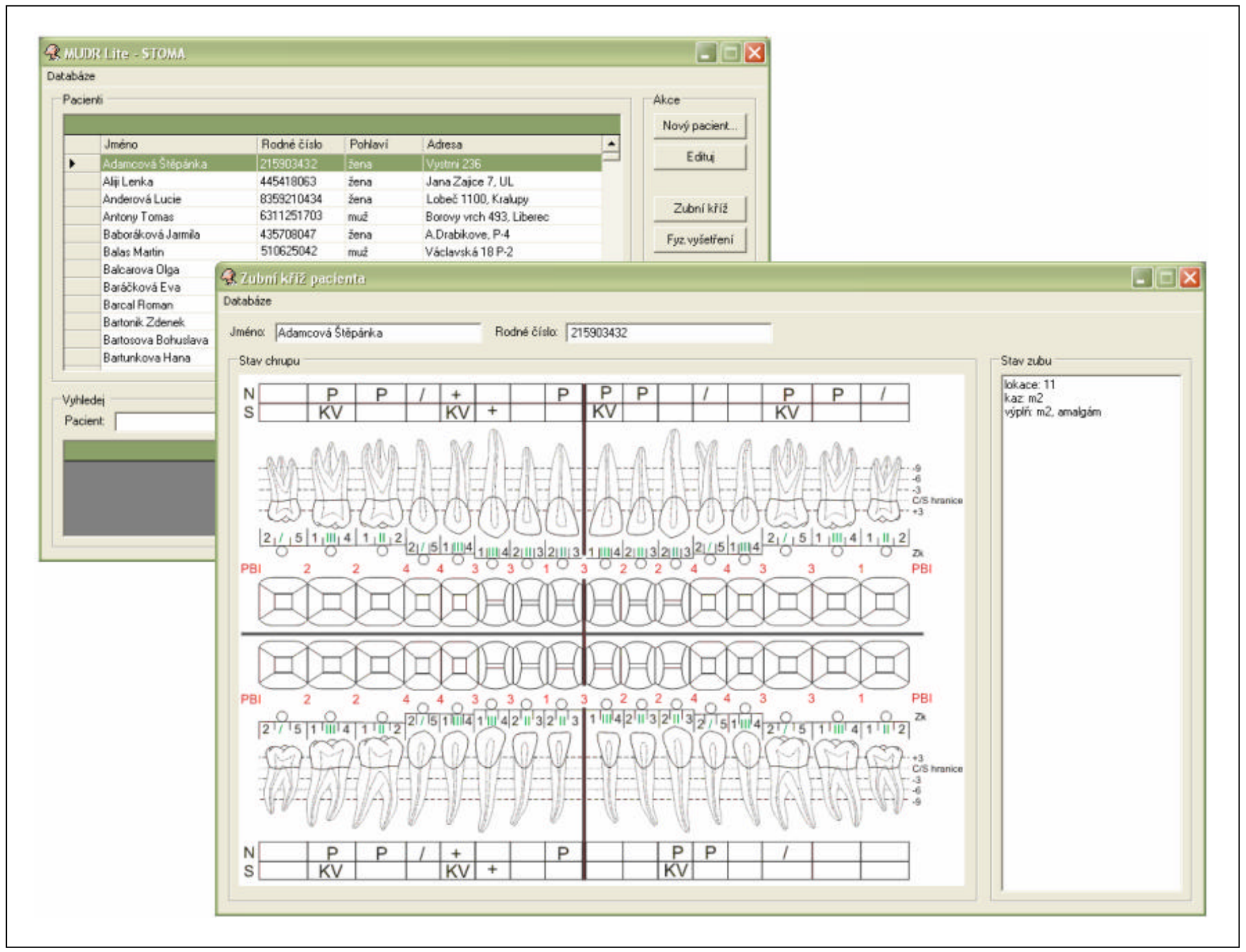

Fig. 3 Application of the MUDR Lite in stomatology

values are in accordance with medical knowledge. The system usually verifies if the input value is inside the defined interval. If a stated diagnosis or a chosen treatment is not in agreement with common medical guidelines, the system warns the user and suggests a diagnosis that is more probable or more appropriate [35],[36],[37],[38].

\section{Condusions}

The ability to manage modern communication tools such as Internet services is one of the most important prerequi- sites for the development and use of eeducation (especially in the area of lifelong education) and e-health applications. Computer literacy and how to prove it is a very important task for today. In medicine and health care, sound education and training plays a very important role in achieving high quality and economy of healthcare. The research in the field of biomedical informatics can strengthen and accelerate the possibility of reaching this goal. Everyone understands that the main source of wealth of any nation is information management and the efficient transformation of information into knowledge. There appear completely new decisive factors for the economics of the near future based on circulation and information exchange. It is clear that modern healthcare cannot be built without information and communication technologies. Telemedicine is largely based on an electronic health record and new methods and tools for how to structure information and extract information from medical free text. Sharing knowledge is the main objective of many research projects. In the paper we described the partial achievements reached by the EuroMISE Center in research and higher education 
supporting better healthcare in future information society.

\section{Acknowledgments}

The work was partly supported by the Research Institutional Plan AV0Z10300504 of the Institute of Computer Science, the Academy of Sciences of the Czech Republic and by the project CZ04307/42011/ 0013.

\section{References}

1. Zvarova J. Education in the Methodology Field of Health Care - EuroMISE, Methods Inf Med 1994; 33(3): 315-7.

2. Zvarova J, Engelbrecht R, van Bemmel JH. Education in Medical Informatics, Statistics and Epidemiology, Int J Med Inf 1997;45(1/2): 3-8.

3. Hasman A, Albert A, Wainwright P, Klar R, Sosa $M$, eds. Education and Training in Health Informatics in Europe. Amsterdam: IOS Press; 1995.

4. IT EDUCTRA CD. Commision of the European Communities; 1998.

5. Zvarova J, Zvara K: Evaluation of Knowledge Using ExaMe Program on the Internet. In: Iakovidis I, Maglaveras N, Trakatellis A, editors. User Acceptance of Health Telematics Applications, Amsterdam: IOS Press; 2000. p. 145-51.

6. Martinkova P, Zvara K jr., Zvarova J, Zvara K. The New Features of the ExaMe Evaluation System and Reliability of its Fixed Tests. Methods Inf Med 2006; 45(3).

7. Martin-Sanchez F, Iakovidis I, Norager S, Maojo V, deGroen P, Van der Lei J, et al. Synergy between Medical Informatics and Bioinformatics: Facilitating Genomic Medicine for Future HealthCare. J Biomed Inform, 2005; 37: 30-42.

8. van Bemmel JH, Zvarova J, editors. Knowledge, Information and Medical Education Amsterdam: North Holland; 1991.

9. Haux R, Leven FJ, Moehr JR, Protti DJ (eds.). Health and Medical Informatics Education. Methods Inf Med 1994 (33)3: 246-331.

10. Hovenga E. Changing Academic Roles: New Approaches to Teaching and Learning. Methods Inf Med. In press 2006.

11. Moehr J. Where to in the Next 10 Years of Health Informatics Education? Methods Inf Med. In press 2006.

12. Zvarova J. Basic Statistics for Biomedical Disciplines. Carolinum, 2000, 2002, 2004, in Czech

13. Zvarova J,Mazura I: Stochastic Genetics, Prague, Carolinum, 2002, in Czech.

14. Bencko V, Hrach K, Maly M, Pikhart H, Reissigova J, Svaeina S, Tomeckova M, Zvarova J. Statistical
Methods in Epidemiology. Volume 1,2, Prague: Carolinum; 2003, in Czech.

15. Zvarova J, Hanzlicek P, Hejl H, Jirkovec Z, Pikhart H, Pøibik V, Smitkova V, Zvara K. Basic Informatics for Biomedicine and Health Care. Prague, Carolinum 2002, in Czech

16. Zvarova J, Svacina S. New Czech Postgraduate Doctoral Program in Biomedical Informatics. In: Surjan G, Engelbrecht R, McNair P, editors. Health Data in the Information Society, Proceedings of MIE 2002. Amsterdam: IOS Press; 2002. p. 766-9.

17. Duplaga M, Zielinski K and Ingram D, editors. Transformation of Healthcare with Information Technologies, Studies in Health Technology and Informatics, Vol 105. Amsterdam: IOS Press; 2004.

18. Zvarova J, Hanzlicek P, Peleska J, Preckova P, Svatek $\mathrm{V}$, Valenta Z, editors. Proceedings of the International Joint Meeting EuroMISE 2004, EuroMISE s.r.o., Prague; 2004.

19. Kaiser K, Miksch S and Tu SW, editors. Computer-based Support for Clinical Guidelines and Protocols Studies in Health Technology and Informatics, Vol 101. Amsterdam: IOS Press; 2004.

20. Zvarova J, Pribik V. Information Society in Czech Healthcare Starting Point to Prognosis for the Year 2013. Int J Med Inf 2002; 66: 59-68.

21. Tomeckova M, Rauch J, Berka P. Guide to Atherosclerosis Risk Factors Data In: Berka P, Cremilleux B, editors. Discovery Challenge 2004. A Collaborative Effort in Knowledge Discovery from Databases. Caen: University of Caen; 2004.

22. Monhart Z. Grunfeldova H, Kadleckova, Jansky P, Zvarova J. Register of Acute Myocardial Infarction - Searching for Generally Applicable Model (in Czech). Supplementum Cor Vasa 2004; 46 (4): 60.

23. Zvarova J: On the Medical Informatics Structure. Int J Med Inf 1997; 44: 75-82.

24. Vajda I, Vesely A, Zvarova J. On the Amount of Information Resulting from Empirical and Theoretical Knowledge. Reviste Matematica Complutense 2005. In press 2005.

25.Zvarova J, Vajda I. On Genetic Information, Diversity and Distance. Methods Inf Med. In press 2005.

26. Semecky J, Zvarova J. On Regular Analysis of Medical Reports. In: Proceedings of NLPBA 2002; Cyprus; 2002. p. 13-6.

27. Antolik J. Automatic Annotation of Medical Records. In: Engelbrecht R, Geissbuhler A, Lovis C, Mihalas G, editors. Connecting Medical Informatics and Bio-Informatics. Proceedings of MIE 2005. Amsterdam: IOS Press; 2005. p. 817-22.

28. Preckova P, Spidlen J, Zvarova J. International Nomenclatures and Metathesauruses in Healthcare. Acta Informatica Medica (submitted).

29. Hanzlicek P, Spidlen J, Nagy M. Universal Electronic Health Record MUDR. In: Duplaga M, Zielinski K, Ingram D, editors. Transformation of Healthcare with Information Technologies. Amster- dam: IOS Press; 2004. p.190-201.

30. Hanzlicek P, Spidlen J, Heroutova H, Nagy M. User Interface of MUDR Electronic Health Record. Int J Med Inform 2005; 74(2-4): 221-7.

31. Spidlen J, Hanzlicek P, Zvarova J. MUDRLite Health Record Tailored to your Particular Needs. In: Duplaga M, Zielinski K, Ingram D, editors. Transformation of Healthcare with Information Technologies. Amsterdam: IOS Press; 2004. p. 202-9.

32. Spidlen J, Hanzlícek P, Riha A, Zvarova J. Flexible Information Storage in MUDR ${ }^{2}$ EHR Int J Med Inf. In press 2006.

33. Teuberova Z, Spidlen J, Pies M, Nagy M, Dostalova T, Hanzlicek P, et al. Interactive Structured Dental Cross, Protetyka Stomatologiczna, A Vision of Modern Prosthodontics EPA 2005, 5; 2005. p.7.

34. Mor P, Aziz B, Samson T, Dongwen W, Omolola $\mathrm{O}$, Quing Z. Guideline Interchange Format 3.5 Technical Spec. Available from: http://smiweb.stanford.edu/projects/intermed-web/guidelines/ GLIF_TECH_SPEC.pdf, InterMed Collaboratory, 2002.

35. Kolesa P, Spidlen J, Zvarova J. Obstacles to Implementing an Execution Engine for Clinical Guidelines Formalized in GLIF. In: Engelbrecht R, Geissbuhler A, Lovis C, Mihalas G, editors. Connecting Medical Informatics and BioInformatics. Proceedings of MIE 2005. Amsterdam: IOS Press; 2005. p. 563-8.

36. Buchtela D, Peleska J, Vesely A, Zvarova J. Method of GLIF Model Construction and Implementation, In: Engelbrecht R, Geissbuhler A, Lovis C, Mihalas $\mathrm{G}$, editors. Connecting Medical Informatics and Bio-Informatics. Proceedings of MIE 2005. Amsterdam: IOS Press; 2005. p.779-84.

37. Zvarova J, Buchtela D, Hanzlicek P, Spidlen J, Vesely A. On Direct Comparing of Medical Guidelines with Electronic Health Record. In: Bubak M, van Albada GD, Sloot P, Dongarra J, editors. Proceedings of ICCS 2004. Berlin: Springer-Verlag. 2004. p. $1133-9$.

38. Buchtela D, Peleska J, Vesely A, Zvarova J. Presentation of Medical Guidelines on a Computer. In: Zielinski K, Duplaga M, Ingram D, editors. Transformation of Healthcare with Information Technologies. Amsterdam: IOS Press; 2004. p. 166-71.

Correspondence to:

Prof. RNDr. Jana Zvarova, Drsc

EuroMISE Centre

Institute of Computer Science AS CR

Pod Vodarenskou vezil 2

18207 Prague 8

Czech Republic

E-mail: zvarova@ euromise.cz 